ARTIGO ORIGINAL

\title{
Tratamento endovascular da reestenose carotídea: resultados em curto prazo
}

\section{Endovascular treatment of carotid artery restenosis: short term Results}

\author{
Ricardo Augusto Carvalho Lujan', Leonardo Aguiar Lucas', Andréia de Fátima Gracio', Giovana Maria Lopes Carvalho², \\ Armando de Carvalho Lobato ${ }^{3}$
}

\section{Resumo}

Contexto: O tratamento cirúrgico da reestenose carotídea apresenta alta taxa de lesão neurológica. Contrariamente, o tratamento endovascular da doença obstrutiva carotídea extracraniana tem se tornado mais factível e gradualmente menores taxas de risco cirúrgico vêm sendo reportadas, tornando-se uma opção em situações especiais, e provavelmente poderá ser considerado o tratamento padrão para reestenose carotídea.

Objetivos: Avaliar a aplicabilidade, a segurança e a eficácia da angioplastia com o uso do stent (ACS) no tratamento da reestenose carotídea (REC) no intraoperatório e no pós-operatório recente (<30 dias).

Métodos: Análise retrospectiva dos pacientes portadores de reestenose carotídea submetidos à angioplastia com stent no período de março 2000 a junho de 2004.

Resultados: Foram analisados 19 pacientes com reestenose carotídea. Quatorze pacientes (74\%) eram do sexo masculino, com média de idade de 74 anos. Quinze (79\%) eram assintomáticos com estenose $>80 \%$, enquanto quatro (21\%) eram sintomáticos com estenose $>70 \%$. Apenas em um paciente não foi utilizado sistema de proteção cerebral. O sucesso técnico foi obtido em todos os casos. Não houve morte ou acidente vascular encefálico no intra ou no pós-operatório recente (30 dias).

Conclusão: $O$ tratamento endovascular da reestenose carotídea mostrou-se uma abordagem factível e segura em curto prazo.

Palavras-chave: Doenças das artérias carótidas; angioplastia com balão; estenose das carótidas.

\begin{abstract}
Context: The surgical treatment of carotid artery restenosis presents a high risk of nerve injury. On the contrary, endovascular treatment for extracranial carotid artery obstructive disease has become more feasible. Gradually, lower rates of surgical risk have been reported, which makes the treatment a good option in special situations. It may be considered as the standard treatment for carotid artery restenosis.

Objective: To evaluate the applicability, safety, and efficacy of the angioplasty with the use of a stent (Carotid Artery Stenting - CAS) for the treatment of carotid artery restenosis, in the intraoperative and early ( $<30$ days) postoperative period.

Methods: Retrospective analysis of patients with carotid artery restenosis who have undergone stenting angioplasty from March 2000 to June 2004.

Results: Nineteen patients with carotid artery restenosis were analyzed. Fourteen (74\%) patients were male, with a mean age of 74 years. Fifteen (79\%) patients were asymptomatic, with stenosis $>80 \%$, whereas 4 (21\%) were symptomatic with stenosis $>70 \%$. In only one patient a cerebral protection system was not used. Technical success was achieved in all cases. There was no death or stroke in the intraoperative or the early postoperative period (30 days).

Conclusion: Endovascular treatment of carotid artery restenosis seems to be a feasible and safe approach in the short term.
\end{abstract}

Keywords: Carotid artery diseases; angioplasty, balloon; carotid stenosis.

Trabalho realizado na Real e Benemerita Associação Portuguesa de Beneficência, São Paulo (SP), Brasil.

Trabalho apresentado no $36^{\circ}$ Congresso Brasileiro de Angiologia e Cirurgia Vascular, realizado em setembro de 2005, Porto Alegre (RS), Brasil.

Especialista em Cirurgia Vascular e Endovascular pela Sociedade Brasileira de Angiologia e Cirurgia Vascular (SBACV) e pela Associação Médica Brasileira (AMB).

${ }^{2}$ Graduanda em Medicina pela Escola Bahiana de Medicina e Saúde Pública, Nazaré/Brotas (BA), Brasil.

Chefe do Instituto de Cirurgia Vascular e Endovascular de São Paulo (ICVE), São Paulo (SP), Brasil.

Não foram declarados conflitos de interesse associados à publicação deste artigo.

Artigo submetido em: 23.08.09. Aceito em: 08.12.10

J Vasc Bras. 2011;10(1):3-8. 


\section{Introdução}

No início dos anos 1990, após a publicação dos resultados dos estudos do North American Symptomatic Carotid Endarterectomy (NASCET) ${ }^{1}$ e do Asymptomatic Carotid Atherosclerosis Study (ACAS) ${ }^{2}$, a endarterectomia carotídea (ECA) ficou estabelecida como padrão-ouro no tratamento cirúrgico da doença obstrutiva carotídea extracraniana de etiologia aterosclerótica; estimando-se, naquele momento, critérios para a intervenção e as respectivas taxas de complicações. O seguimento no pós-operatório da ECA associado ao avanço e ao uso mais abrangente dos testes diagnósticos não invasivos, em especial a ultrassonografia com Doppler, proporcionou um aumento no número de diagnóstico de pacientes portadores de reestenose da artéria carótida pósendarterectomia ${ }^{3}$.

Stoney e String ${ }^{4}$ reportaram a primeira abordagem cirúrgica para tratamento da reestenose carotídea pós-endarterectomia. Posteriormente, diversos autores apresentaram resultados satisfatórios da endarterectomia na reestenose carotídea com taxa de sobrevida livre de novos eventos neurológicos de $92 \%$ e de reestenose severa de $89 \%{ }^{5}$. Entretanto, permanece alta a taxa de morbimortalidade da intervenção cirúrgica na reestenose carotídea ${ }^{6}$. Dessa forma, o tratamento endovascular surge como uma opção cirúrgica à ECA nos pacientes portadores reestenose carotídea ${ }^{7}$.

Sendo assim, reportamos a experiência do Instituto de Cirurgia Vascular e Endovascular de São Paulo (ICVE-SP) no tratamento endovascular da reestenose carotídea.

\section{Objetivo}

Avaliar a aplicabilidade, a segurança e a eficácia da angioplastia com o uso do stent (ACS) no tratamento da reestenose carotídea (REC) no intraoperatória e no pósoperatório recente ( $<30$ dias).

\section{Método}

De março de 2000 a junho de 2004 foram analisados, de forma retrospectiva, os prontuários de 19 pacientes portadores de reestenose carotídea pós-endarterectomia há pelo menos dois anos e submetidos à angioplastia com stent. Todos foram consecutivos e o critério de seleção foi reestenose; portanto, não houve exclusão. Os pacientes foram operados previamente em outros serviços e a procura pelo Instituto ocorreu por orientação do médico ou livre demanda. Todos os indivíduos foram avaliados previamente à intervenção com ultrassonografia com Doppler a cores e angiorressonância nuclear magnética. A angiografia digital foi realizada apenas no intraoperatório. Além disso, foram utilizados os critérios do NASCET para mensuração do grau de estenose.

A terapêutica antiplaquetária foi realizada com clopidogrel (75 mg/dia) iniciado 4 dias antes do procedimento ou dose ataque de $300 \mathrm{mg}$ na véspera do procedimento e mantido no pós-operatório por pelo menos 30 dias. Os pacientes que não vinham em uso de ácido acetilsalicílico eram orientados a usá-lo independentemente do tempo antes da reintervenção e mantê-lo após o procedimento de forma contínua. No intraoperatório, após confirmação arteriográfica da lesão, era administrada heparina não fracionada (5.000 UI) em bolus endovenoso. Doses adicionais eram administradas caso houvesse evidência de formação de coágulo no intraoperatório ou o tempo de coagulação ativada (TCA) não estivesse adequado. A monitorização da coagulação era realizada entre 5 a 10 minutos após administração da heparina não fracionada em bolus, com nova coleta caso o procedimento se prolongasse por mais de 2 horas. Uso de atropina foi restrito aos pacientes que apresentaram bradicardia (<60 bpm) sintomática.

Todos os procedimentos foram realizados por punção da artéria femoral comum, com anestesia local (lidocaína $1 \%$ sem vasoconstrictor), sob cuidado anestésico monitorado. O sistema de proteção cerebral, tipo microporos, foi utilizado na maioria dos pacientes. Além disso, foram empregados apenas stents autoexpansíveis de nitinol.

Os pacientes pernoitaram no pós-operatório imediato em unidade de terapia intensiva como de rotina.

Ataque isquêmico transitório (AIT) foi definido como déficit neurológico focal de início súbito, com regressão completa dos sintomas dentro de 24 horas. Acidente vascular encefálico (AVE) no pós-operatório foi definido como déficit neurológico focal agudo que permanecesse por mais de 24 horas e estivesse correlacionado com mudança no estudo de imagem cerebral. Ambos diagnósticos eram estabelecidos por neurologistas independentes.

O sucesso técnico foi definido como estenose residual menor que $30 \%$.

\section{Resultados}

No período do estudo foram realizadas angioplastias com stent em 19 pacientes portadores de reestenose carotídea. A média de idade foi de 74 anos, com $74 \%(n=14)$ do sexo masculino. Os pacientes assintomáticos $(79 \%, n=15)$ apresentavam estenoses $>80 \%$, enquanto os sintomáticos $(21 \%, \mathrm{n}=4)$ apresentavam lesões $>70 \%$, de acordo com os critérios estabelecidos pelo NASCET. As lesões foram localizadas, em $71 \%(n=13)$ dos casos, na artéria carótida 
direita. Todos os pacientes haviam sido submetidos à endarterectomia carotídea há mais de dois anos. Como foram encaminhados, não havia condições de saber se apresentavam remendo ou não. As comorbidades estão descritas no Tabela 1.

Todos os procedimentos foram realizados por punção da artéria femoral comum sob anestesia local. Não foram observadas fístulas arteriovenosas iatrogênicas, pseudoaneurismas ou qualquer outra situação clínica que necessitasse de abordagem cirúrgica no sítio de punção durante o período de acompanhamento dos pacientes. A taxa de sucesso técnico foi de $100 \%$. Os stents autoexpansíveis de nitinol foram utilizados em todos os casos, e, exceto um paciente, os demais fizeram uso de sistema de proteção cerebral. Em nenhum dos casos foi encontrado debris macroscópicos no sistema de proteção cerebral. Espasmo da artéria carótida interna ocorreu em 8 pacientes (47\%), os quais utilizaram o sistema de proteção cerebral. Todos os casos de espasmo carotídeo foram tratados com a retirada do sistema de proteção cerebral. Não houve óbito, acidente vascular encefálico e/ou ataque isquêmico transitório no intraoperatório ou no pós-operatório recente.

\section{Discussão}

A endarterectomia da artéria carótida (ECA) permanece como padrão-ouro no tratamento da doença obstrutiva carotídea ${ }^{1,2,8}$. Os benefícios cirúrgicos da ECA são relacionados à baixa taxa de morbimortalidade associado à prevenção de eventos neurológicos em longo prazo $^{9,10}$. Contudo, a intervenção cirúrgica não evita a reestenose por hiperplasia miointimal (curto/médio prazo) ou por progressão da aterosclerose (longo prazo). Estudos baseados em testes diagnósticos não invasivos reportam taxas de reestenose carotídea sintomática pós-endarterectomia variando em torno de 0,6 a 3,6\% e assintomática variando de 8,8 a $19 \%{ }^{11}$. Na

Tabela 1 - Dados demográficos

\begin{tabular}{lll}
\hline & $\mathrm{n}$ & $\%$ \\
\hline Sexo masculino* $^{*}$ & 14 & 73,7 \\
Comorbidades* $^{*}$ & \\
DAC & 12 & 63,2 \\
HAS & 10 & 52,6 \\
DM & 5 & 26,3 \\
DPOC & 4 & 21,1 \\
ICC & 2 & 10,5 \\
IRC & 1 & 5,3 \\
\hline
\end{tabular}

*Dados expressos como frequência (n) e respectivas percentagens (\%); DAC: doença arterial coronariana; HAS: hipertensão arterial sistêmica; DM: Diabetes mellitus; DPOC: doença pulmonar obstrutiva crônica; ICC: insuficiência cardíaca congestiva; IRC: insuficiência renal crônica. amostragem, todos os pacientes haviam sido submetidos à endarterectomia carotídea há mais de dois anos (Figura 1A, $1 \mathrm{~B}$ e $1 \mathrm{C})$.

Após a publicação de Stoney e String ${ }^{4}$, o tratamento para reestenose carotídea sintomática ou de alto grau $(>80 \%)$ tem sido a reoperação da artéria carótida. Entretanto, a reintervenção cirúrgica nesse território além de ser tecnicamente mais difícil, proporciona riscos adicionais, dentre os quais lesão de nervo craniano e complicações relacionadas à ferida operatória. A proposta da angioplastia com stent (ACS) na doença carotídea obstrutiva extracraniana é atraente tanto para o cirurgião

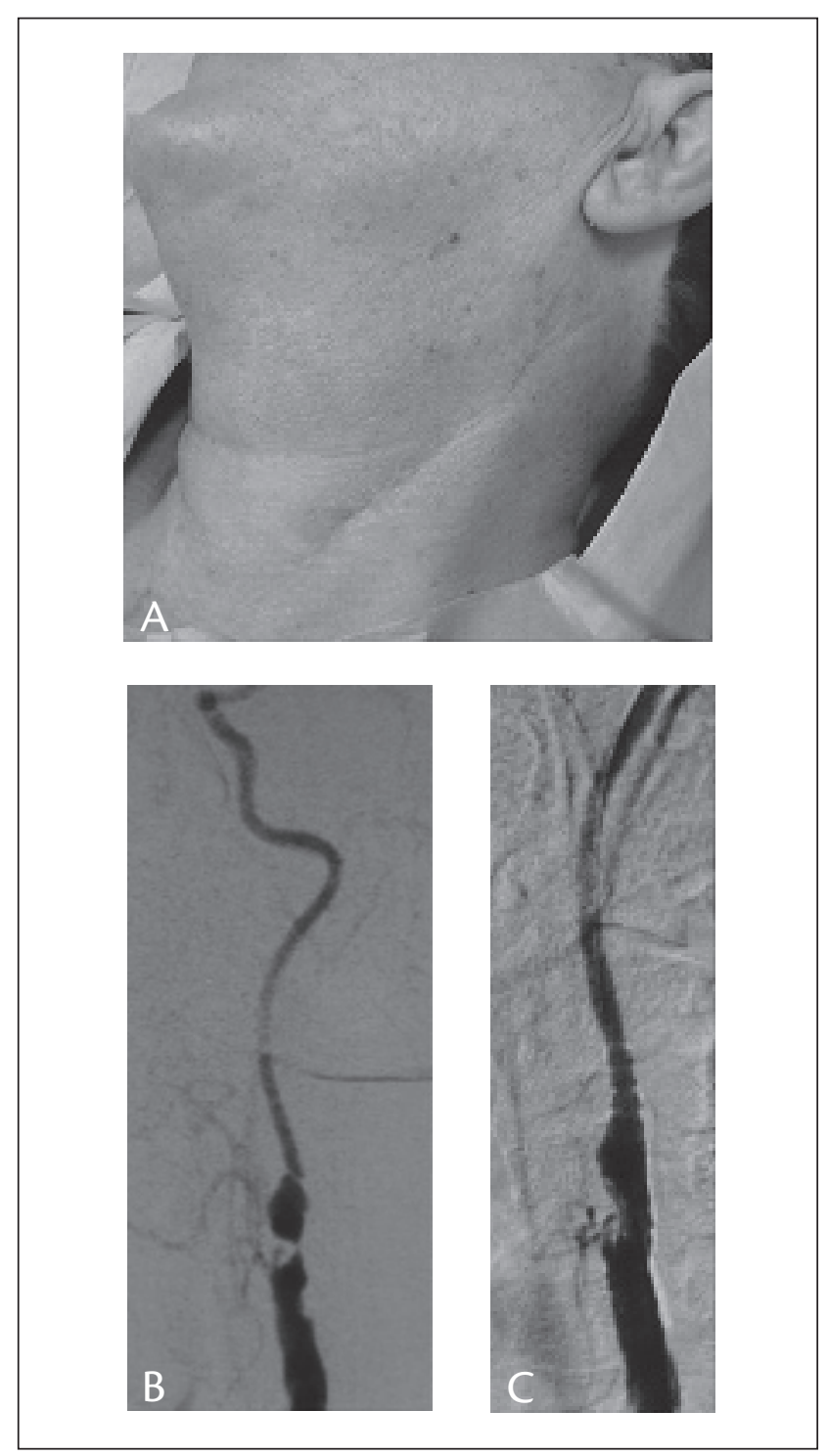

Figura 1 - (A) Paciente no pós-operatório tardio de endarterectomia carotídea esquerda. (B) Angiografia diagnóstica demonstrando estenose crítica. (C) Angiografia de controle pós-angioplastia com stent apresentando resultado satisfatório 
vascular quanto para o paciente por ser minimamente invasiva, apresentar baixa taxa de complicações relacionadas à ferida operatória, uma provável redução no tempo de hospitalização, além de, teoricamente, evitar anestesia geral e incisão cervical (não apresentando risco de lesão de nervos cranianos).

Em 2005, de Borst et al. ${ }^{10}$ reportaram os resultados obtidos de 57 angioplastias carotídeas realizadas em 55 pacientes que apresentavam reestenose carotídea pós-ECA acompanhados durante 4 anos. Além disso, observaram uma taxa de sobrevida livre de reestenose pós-ACAS de 93, 85,82 e $76 \%$ em 1, 2, 3 e 4 anos de seguimento, respectivamente. Nesse trabalho, os pacientes que apresentaram reestenose intra-stent de alto grau assintomático $(\geq 80 \%)$ ou sintomático $(\geq 70 \%)$ foram submetidos a uma re-intervenção, onde em $50 \%$ dos casos foi optado por reabordagem via percutânea, e nos demais, uma nova ECA com remoção do stent foi realizada. Apenas em um caso houve uma terceira angioplastia. Todos estes pacientes não apresentaram novos eventos neurológicos após a re-intervenção durante o seguimento do estudo. É interessante notar que, de acordo com o que foi reportado por de Borst et al., o tratamento endovascular na reestenose carotídea apresentou alta taxa de sucesso técnico e clínico, além de não inviabilizar uma reabordagem tanto cirúrgica quanto por via percutânea nos casos de reestenose intra-stent de alto grau. Ademais, em ambas as situações houve sucesso clínico por tempo prolongado após a reintervenção.

Hobson et al. ${ }^{12}$ apresentaram resultados combinados (morbidade e/ou mortalidade neurológica) perioperatórios em 30 dias, nos quais 16 pacientes foram submetidos a angioplastia com stent na reestenose carotídea comparados com outros 16 pacientes submetidos à reoperações convencionais. Não houve AVE e/ou óbito em ambos os grupos, porém o grupo submetido à cirurgia convencional apresentou taxa de 6,2\% de lesão de nervos. É fato relevante que apesar dos resultados comparativos semelhantes, neste estudo, nem todos pacientes submetidos a ACS utilizaram o sistema de proteção cerebral.

Reimers et al. ${ }^{13}$ demonstraram a presença de debris de placas ateroscleróticas retidos no sistema de proteção cerebral (SPC) em mais de 50\% dos pacientes submetidos a angioplastia com stent da artéria carótida. O potencial de microembolização da placa também foi observado por Garami et al. ${ }^{14}$, com o uso do Doppler trans-craniano, durante a ACS. Os autores concluiram que o uso do SPC reduz, porém não elimina, a microembolização cerebral durante a ACS. Em nossa casuística, exceto um paciente, todos os demais utilizaram o SPC, porém não foram observados debris macroscópicos nos sistemas de proteção cerebral

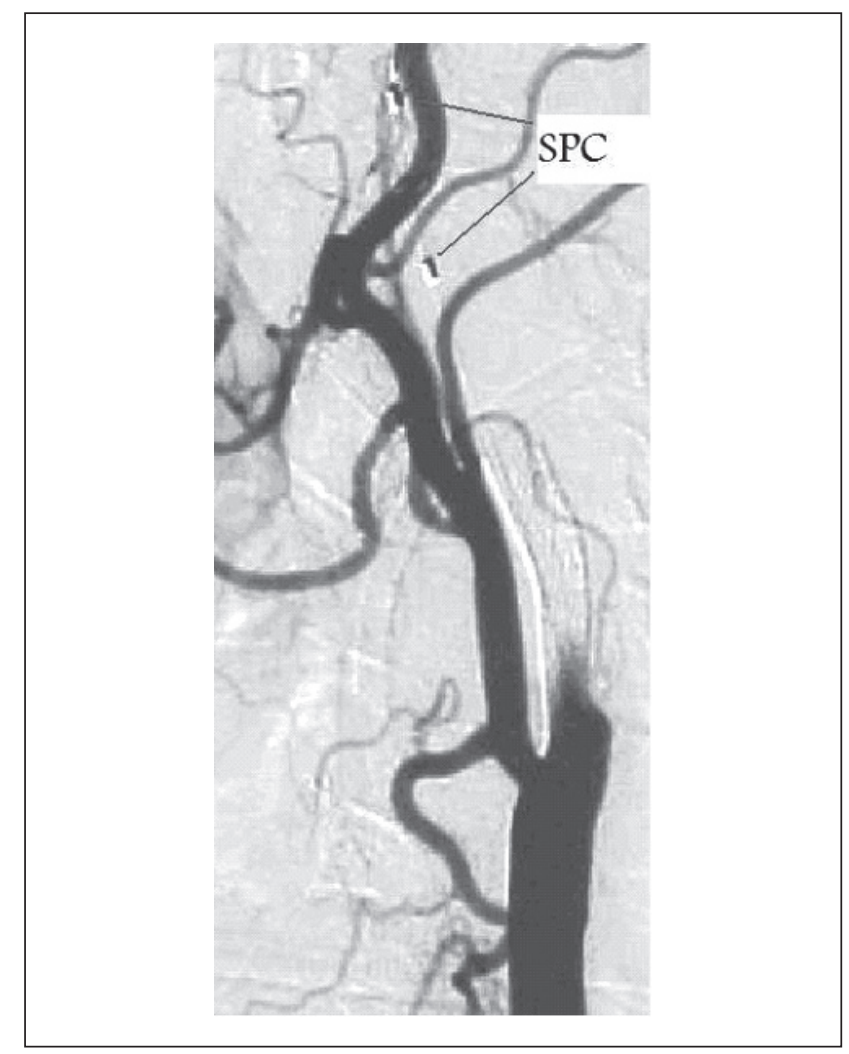

Figura 2 - Espasmo artéria carótida interna. Notar a presença do sistema de proteção cerebral. SPC: sistema de proteção cerebral.

utilizados. Contudo, a alta taxa de espasmo da artéria carótida interna (Figura 2) chamou-nos atenção quanto ao uso do sistema de proteção cerebral, o qual demonstrou não ser um dispositivo inocúo, podendo levar a complicações maiores. Semelhante à literatura, a simples remoção do SPC reverteu o espasmo carotídeo, sem apresentar repercussão neurológica ${ }^{15}$. Esse resultado também foi observado por Roffi et al. ${ }^{16}$ ao estudarem o comportamento dos diferentes tipos de SPC.

Os avanços da tecnologia como menor perfil dos stents e melhores dispositivos de SPC, associado ao crescente manuseio, conhecimento e domínio dessa técnica tem adicionado segurança ao tratamento endovascular e proporcionado melhores resultados nos últimos anos. Entretanto, limitações a técnica ainda existem. Chong et al. ${ }^{17}$ estudaram 177 pacientes portadores de doença carotídea obstrutiva extracraniana em programação de intervenção. Observaram que em 113 pacientes era possível a cirurgia endovascular, enquanto outros 64 eram preferíveis a endarterectomia. Critérios anatômicos foram os principais responsáveis pela não eligibilidade da angioplastia, dos quais a tortuosidade carotídea e doença arterial proximal correspoderam a $70 \%$ dos casos. Por 
outro lado, em 51 pacientes, a angioplastia carotídea foi considerada pelo autor como preferível por apresentarem pescoço hóstil devido à radioterapia cervical ou à endarterectomia carotídea prévia, comorbidades significativas ou pacientes em programação de revascularização miocárdica.

Apesar dos diversos estudos randomizados publicados, até o momento, não existem argumentos com nível um de evidência a favor ou contra a ACS em relação à ECA na doença carotídea obstrutiva extracraniana sintomática ou assintomática, incluindo na $\mathrm{REC}^{18}$. Bettendorf et al. ${ }^{7}$ relataram em seu trabalho que ACS é um método seguro e efetivo para tratar pacientes com REC e pode se tornar o tratamento de escolha. Outros autores ${ }^{11,19}$ têm indicado a ACS nos pacientes considerados de alto risco ou não eligivéis à $\mathrm{ECA}$, baseados nos critérios adotados pelos estudos ACAS e NASCET. Gurm et al..$^{20}$ publicaram os resultados do estudo SAPPHIRE reportando a não inferioridade da ACS em relação à ECA em pacientes de alto risco, em curto e longo prazo. Relatou ainda taxa de reintervenção de $1 \%$ por ano no grupo submetido à angioplastia contra $4 \%$ por ano no grupo submetido à ECA nos primeiros três anos de seguimento. Em nosso estudo indicamos ACS na REC em pacientes sintomáticos apresentando lesão $\geq 70 \% \mathrm{e}$, nos assintomáticos com estenose $\geq 80 \%$, seguindo a literatura contemporânea ${ }^{11}$.

Como publicado anteriormente ${ }^{21}$, no período do estudo adotávamos como rotina o uso de ultrassonografia com Doppler colorido, como exame de escolha no segmento pós-operatório de pacientes submetidos à ECA. Havendo evidência de lesão hemodinamicamente significativa, prosseguíamos a investigação com a angioressonância nuclear magnética. Atualmente substituímos essa última pela angiotomografia multi-slice, por possuir uma boa acurácia referente ao grau de estenose, possibilidade de reconstrução tridimensional, apresentação similar à arteriografia, além de ser um método não invasivo ${ }^{22}$. A arteriografia diagnóstica persiste como padrão-ouro, entretanto mantemos a preferência de realizar esse procedimento apenas no intraoperatório.

\section{Conclusão}

Angioplastia com stent na reestenose carotídea demostrou ser um procedimento com resultado técnico satisfatório em curto prazo, com baixa taxa de morbimortalidade operatória, além de ausência de lesão de nervos cranianos. Estudos randomizados com casuísticas maiores e seguimento em longo prazo são necessários para validar a ACS como tratamento padrão para REC.

\section{Referências}

1. [No authors listed]. Beneficial effect of carotid endarterectomy in symptomatic patients with high-grade stenosis. North American Symptomatic Carotid Endarterectomy Trial Collaborators. N Engl J Med. 1991 Aug 15;325:445-53.

2. [No authors listed]. Endarterectomy for asymptomatic carotid artery stenosis. Executive Committee for the Asymptomatic Carotid Atherosclerosis Study. JAMA. 1995;273:1421-8.

3. Jahromi AS, Cinà CS, Liu Y, Clase CM. Sensitivity and specificity of color duplex ultrasound measurement in the estimation of internal carotid artery stenosis: a systematic review and meta-analysis. J Vasc Surg. 2005;41:962-72.

4. Stoney RJ, String ST. Recurrent carotid stenosis. Surgery. 1976; 80:705-10.

5. Cho JS, Pandurangi K, Conrad MF, et al. Safety and durability of redo carotid operation: an 11-year experience. J Vasc Surg. 2004;39:155-61.

6. Bowser AN, Bandyk DF, Evans A, et al. Outcome of carotid stentassisted angioplasty versus open surgical repair of recurrent carotid stenosis. J Vasc Surg. 2003;38:432-8.

7. Bettendorf MJ, Mansour MA, Davis AT, et al. Carotid angioplasty and stenting versus redo endarterectomy for recurrent stenosis. Am J Surg. 2007;193:356-9; discussion 359.

8. Barnett HJ, Taylor DW, Eliasziw M, et al. Benefit of carotid endarterectomy in patients with symptomatic moderate or severe stenosis. North American Symptomatic Carotid Endarterectomy Trial Collaborators. N Engl J Med. 199812;339:1415-25.

9. Young B, Moore WS, Robertson JT, et al. An analysis of perioperative surgical mortality and morbidity in the asymptomatic carotid atherosclerosis study. ACAS Investigators. Asymptomatic Carotid Artheriosclerosis Study. Stroke. 1996; 27:2216-24.

10. de Borst GJ, Ackerstaff RG, de Vries JP, et al. Carotid angioplasty and stenting for postendarterectomy stenosis: long-term follow-up. J Vasc Surg. 2007; 45:118-23.

11. Lal BK, Hobson RW 2nd. Management of carotid restenosis. J Cardiovasc Surg (Torino). 2006;47:153-60.

12. Hobson RW 2nd, Goldstein JE, Jamil Z, et al. Carotid restenosis: operative and endovascular management. J Vasc Surg. 1999;29:22835; discussion 235-8.

13. Reimers B, Corvaja N, Moshiri S, et al. Cerebral protection with filter devices during carotid artery stenting. Circulation. 2001;104:12-5.

14. Garami ZF, Bismuth J, Charlton-Ouw KM, et al. Feasibility of simultaneous pre- and postfilter transcranial Doppler monitoring during carotid artery stenting. I Vasc Surg. 2009;49:340-4, 345.e1-2; discussion 345.

15. Powell RJ, Alessi C, Nolan B, et al. Comparison of embolization protection device-specific technical difficulties during carotid artery stenting. J Vasc Surg. 2006;44:56-61.

16. Roffi $M$, Greutmann $M$, Schwarz $U$, et al. Flow impairment during protected carotid artery stenting: impact of filter device design. J Endovasc Ther. 2008;15:103-9.

17. Chong PL, Salhiyyah K, Dodd PD. The role of carotid endarterectomy in the endovascular era. Eur J Vasc Endovasc Surg. 2005;29:597-600.

18. Bonamigo TP, Lucas ML. Análise crítica das indicações e resultados do tratamento cirúrgico da doença carotídea. J Vasc Bras. 2007;6:366-77. 
19. Veith FJ, Amor M, Ohki T, et al. Current status of carotid bifurcation angioplasty and stenting based on a consensus of opinion leaders. J Vasc Surg. 2001;33:S111-6.

20. Gurm HS, Yadav JS, Fayad P, Katzen BT, Mishkel GJ, Bajwa TK, Ansel G, Strickman NE, Wang H, Cohen SA, Massaro JM, Cutlip DE; SAPPHIRE Investigators. Long-term results of carotid stenting versus endarterectomy in high-risk patients. N Engl J Med. 2008;358:1572-9.

21. Lujan RAC, Lucas LA, Gracio AF, at al. Tratamento endovascular da doença obstrutiva carotídea em pacientes de alto risco: resultados imediatos. J Vasc Bras. 2006;5:23-29.

22. Vanhoenacker PK, Decramer I, Bladt O, et al. Multidetector computed tomography angiography for assessment of in-stent restenosis: meta-analysis of diagnostic performance. BMC Med Imaging. 2008;8:14
Correspondência: Ricardo Augusto Carvalho Lujan Avenida Princesa Leopoldina, 914, sala 309 - Barra Avenida CEP: 40144-900 - Salvador (BA), Brasil Tel./Fax: (71) 3336-9882

Contribuições dos autores Concepção e desenho do estudo: RACL, AFG, ACL Análise e interpretação dos dados: RACL, AFG, LAL, ACL Coleta de dados: $A C L$ Redação do artigo: RACL, GMLC Revisão crítica do texto: RACL, AFG, $A C L$ Aprovação final do artigo*: RACL, LAL, AFG, GMLC, ACL Análise estatística: RACL, LAL Responsabilidade geral pelo estudo: RACL, LAL, $A C L$ Informações sobre financiamento: N/A *Todos os autores leram e aprovaram a versão final submetida ao I Vasc Bras. 